\title{
Simvastatin rescues homocysteine-induced apoptosis of osteocytic MLO-Y4 cells by decreasing the expressions of NADPH oxidase 1 and 2
}

\author{
Ayumu Takeno, Ippei Kanazawa, Ken-ichiro Tanaka, Masakazu Notsu, Maki Yokomoto-Umakoshi and \\ Toshitsugu Sugimoto
}

Department of Internal Medicine 1, Shimane University Faculty of Medicine, Izumo 693-8501, Japan

\begin{abstract}
Clinical studies have shown that hyperhomocysteinemia is associated with bone fragility. Homocysteine (Hcy) induces apoptosis of osteoblastic cell lineage by increasing oxidative stress, which may contribute to Hcy-induced bone fragility. Statins, 3-hydroxy-3-methylglutaryl coenzyme A reductase inhibitors, ameliorate oxidative stress by regulating oxidant and anti-oxidant enzymes. However, the effects of statins on Hcy-induced apoptosis of osteocytes are unknown. This study was thus aimed to investigate whether or not statins prevent Hcy-induced apoptosis of osteocytic MLO-Y4 cells and regulate NADPH oxidase (Nox) expression. TUNEL staining showed that $5 \mathrm{mM}$ Hcy induced apoptosis of MLO-Y4 cells, and that co-incubation of $10^{-9}$ or $10^{-8} \mathrm{M}$ simvastatin significantly suppressed the apoptotic effect. Moreover, we confirmed the beneficial effect of simvastatin against Hcy's apoptotic effect by using a DNA fragment ELISA assay. However, TUNEL staining showed no significant effects of pravastatin, a hydrophilic statin, on the Hcy-induced apoptosis. Real-time PCR showed that Hcy increased the mRNA expressions of Nox1 and Nox2, whereas simvastatin inhibited the stimulation of Nox1 and Nox2 expressions by Hcy. In contrast, neither Hcy nor simvastatin had any effect on Nox 4 expression. These findings indicate that simvastatin prevents the detrimental effects of Hcy on the apoptosis of osteocytes by regulating the expressions of Nox 1 and Nox2, suggesting that statins may be beneficial for preventing Hcy-induced osteocyte apoptosis and the resulting bone fragility.
\end{abstract}

Keywords: Osteocyte, Simvastatin, Homocysteine, Apoptosis, Statin

STATIN is an inhibitor of 3-hydroxy-3-methylglutaryl coenzyme A (HMG-CoA) reductase, which acts as a rate-limiting enzyme for endogenous cholesterol synthesis and is considered a key enzyme in the mevalonate pathway, and it is widely used to prevent cardiovascular events [1-4]. Statin has recently been shown to exert pleiotropic effects on various cells, which may not be directly related to cholesterol synthesis [5]. Since the crucial roles of the mevalonate pathway in bone forming cells such as osteoblasts [6-8] and osteoclasts [9] were reported, the agents inhibiting HMGCoA reductase have also attracted attention in the field of bone research. Statin induces bone morphogenetic

Submitted Aug. 12, 2015; Accepted Jan. 6, 2016 as EJ15-0480 Released online in J-STAGE as advance publication Feb. 3, 2016 Correspondence to: Ippei Kanazawa, M.D., Ph.D., Department of Internal Medicine 1, Shimane University Faculty of Medicine, 891, Enya-cho, Izumo, Shimane, 693-8501, Japan.

E-mail: ippei.k@med.shimane-u.ac.jp

(c) The Japan Endocrine Society protein-2 (BMP-2) expression in osteoblasts and stimulates bone formation $[10,11]$ as well as promotes mineralization $[7,12]$ and suppresses apoptosis in osteoblastic MC3T3-E1 cells [13].

Previous clinical studies have suggested that elevated plasma homocysteine (Hcy) level is associated with osteoporotic fracture [14-17]. However, the mechanisms underlying Hcy-induced bone fragility have yet to be completely elucidated. We previously examined the effects of Hcy on apoptosis of MLO-Y4 cells, a mouse osteocyte cell line, and showed that Hcy dose-dependently induced the apoptosis of the cells via increasing NADPH oxidase (Nox) 1 and Nox2 [18], which are oxidative stress inducing enzymes $[19,20]$, suggesting that osteocyte apoptosis by oxidative stress may be one of the underlying mechanisms of Hcy-induced bone fragility. On the other hand, many studies have demonstrated that statins ameliorate oxidative stress by regulating Nox expression 
and/or activity in various cell types [21-24], including osteoblasts $[25,26]$. Therefore, we hypothesized that statins might prevent Hcy-induced apoptosis. To date, however, there are no reports investigating the effects of statins on osteocytes.

\section{Materials and Methods}

\section{Reagents}

Cell culture medium and supplements were purchased from GIBCO-BRL (Rockville, MD). Simvastatin, pravastatin and DL-homocysteine were purchased from Sigma-Aldrich (St. Louis, MO). All other chemicals were of the highest grade commercially available.

\section{Cell cultures}

MLO-Y4, a murine long bone-derived osteocytic cell line, was kindly provided by Dr. Lynda F. Bonewald, University of Missouri-Kansas City. Cells were cultured on collagen-coated plates in $\alpha$-minimum essential medium ( $\alpha$-MEM) supplemented with $10 \%$ fetal bovine serum (FBS) and 1\% penicillin-streptomycin in $5 \% \mathrm{CO}_{2}$ at $37^{\circ} \mathrm{C}$. The medium was changed twice a week, and the cells were passaged when they were $80 \%$ confluent.

\section{TUNEL staining}

Apoptosis of MLO-Y4 cells was assessed by TUNEL staining using an in situ cell death detection kit (Roche, Germany) according to the manufacturer's protocol. Briefly, the cells were incubated in chamber slides. After the cells were confluent, agents such as Hcy, simvastatin and pravastatin were added at their specified concentrations and the cells were incubated for $72 \mathrm{~h}$. Fixation, blocking, and permeabilization were then performed according to the manufacturer's protocol. Slides were immersed in TUNEL reaction mixture for $60 \mathrm{~min}$ at $37^{\circ} \mathrm{C}$ in a humidified atmosphere in the dark. Slides were analyzed using optical microscopy. The average value of TUNEL-positive cells in one microscopic field $(200 \times)$ was used to evaluate the degree of apoptosis. TUNEL-positive cells were counted in six or eight randomly selected areas of TUNEL-stained slides, and the average value was calculated.

\section{Apoptosis assay using a DNA fragment detection ELISA kit}

Apoptosis was assessed by using Cell Death
Detection ELISA ${ }^{\text {PLUS }}$ kit (Roche Applied Science, Indianapolis, IN) according to the manufacturer's protocol. Cells were incubated in 96-well plates, and Hcy and/or simvastatin were added at their specified concentrations after the cells were confluent. After incubation for $60 \mathrm{~h}$, the cells were lysed using $200 \mu \mathrm{L}$ of lysis buffer. After centrifugation, $20 \mu \mathrm{L}$ of the supernatant was transferred to a streptavidin-coated microplate and exposed to anti-histone antibody (biotin-labeled) and anti-DNA antibody (peroxidase-conjugated) for $2 \mathrm{~h}$ at room temperature. Each well was washed 3 times with the incubation buffer, and antibody-nucleosome complexes bound to the microplate were determined spectrophotometrically using 2,2'-azino-bis (3-ethylbenzothiazoline-6-sulphonic acid) (ABTS). Absorbance measured using the kit was proportional to the degree of apoptosis. Results were expressed relative to the control.

\section{Quantification of gene expression using real-time PCR}

We used SYBR green chemistry to determine the mRNA levels of Nox1, Nox2, Nox4 and a housekeeping gene, 36B4. The primer sequences were: Nox1 forward, 5'-ATGCCCCTGCTGCTCGAATA-3' and Nox1 reverse, 5'-AAATTGCCCCTCCATTTCCT-3'; Nox2 forward, 5'-ACCGCCATCCACACAATTG-3', Nox2 reverse, 5'-CCGATGTCAGAGAGAGCTATTGAA-3'; Nox4 forward, 5'-CTGCATCTGTCCTGAACCTCAA-3', Nox4 reverse, 5'-TCTCCTGCTAGGGACCTTCTGT-3'; 36B4 forward, 5'-AAGCGCGTCCTGGCATTGTCT-3', 36B4 reverse, 5'-CCGCAGGGGCAGCAGTGGT-3'. Real-time PCR was performed using $1 \mu \mathrm{L}$ of cDNA in a $25 \mu \mathrm{L}$ reaction volume with ABI PRISM 7000 (Applied Biosystems, Waltham, MA). The doublestranded DNA-specific dye SYBR Green I was incorporated into the PCR buffer provided in the SYBR Green Real-time PCR Master Mix (Toyobo Co. Ltd., Tokyo, Japan) to enable quantitative detection of the PCR product. The PCR conditions were $95^{\circ} \mathrm{C}$ for 15 min, 40 cycles of denaturation at $94^{\circ} \mathrm{C}$ for $15 \mathrm{~s}$, and annealing and extension at $60^{\circ} \mathrm{C}$ for $1 \mathrm{~min}$. $36 \mathrm{~B} 4$ was used to normalize the differences in the efficiencies of reverse transcription.

\section{Statistics}

Results are expressed as means \pm standard error (SE). Statistical evaluations for differences between groups were performed using one-way ANOVA fol- 
lowed by Fisher's protected least significant difference. For all statistical tests, a $p$ value $<0.05$ was considered statistically significant.

\section{Results}

\section{Simvastatin prevented Hcy-induced apoptosis of MLO-Y4 cells}

The effects of Hcy and simvastatin on apoptosis of MLO-Y4 cells were examined using TUNEL staining (Fig. 1a and 1b). As previously reported, incubation with $5 \mathrm{mM}$ Hcy significantly increased apoptosis of MLO-Y4 cells $(p<0.001)$ [18]. Co-incubation of $10^{-9}$ or $10^{-8} \mathrm{M}$ simvastatin significantly suppressed Hcy's apoptotic effect ( $p<0.05$ and $p<0.001$, respectively), whereas $10^{-8} \mathrm{M}$ simvastatin alone had no effect on apoptosis of the cells. Moreover, we confirmed by using a DNA fragment detection ELISA kit that Hcyinduced apoptosis was significantly inhibited by treatments with $10^{-8} \mathrm{M}$ simvastatin $(p<0.01)$ (Fig. 2).

Next, we examined the effects of pravastatin, a hydrophilic statin, on Hcy-induced apoptosis of MLOY4 cells. TUNEL staining showed that pravastatin had a tendency to suppress Hcy-induced apoptosis (Fig. 3a). However, the difference was not significant by quantification of cell count (Fig. 3b).

\section{Simvastatin suppressed upregulation of Hcy-induced Nox1 and Nox2}

We examined the effects of simvastatin on Hcyinduced Nox1 and Nox 2 mRNA expressions in this study. As shown in Fig. 4a and 4b, Hcy significantly increased mRNA expressions of Nox1 and Nox2 $(p<0.001)$, while co-incubation of $10^{-9}$ and $10^{-8} \mathrm{M}$ simvastatin significantly inhibited the Hcy-induced upregulation of Nox1 $(p<0.05)$, and $10^{-8} \mathrm{M}$ simvastatin significantly inhibited the upregulation of Nox2 $(p<0.05)$. On the other hand, neither Hcy nor simvastatin affected the expression of Nox4 (Fig. 4c).

\section{Discussion}

In this study, we confirmed that Hcy increased the expression of Nox 1 and Nox 2 as well as induced the apoptosis of osteocytic MLO-Y4 cells as we previously reported [18]. In addition, we showed for the first time that simvastatin ameliorated the Hcy-induced apoptosis and the upregulation of Nox1 and Nox2 in MLO-Y 4 cells. a

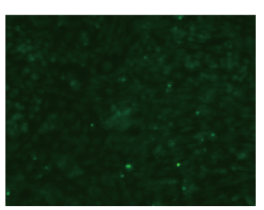

Control

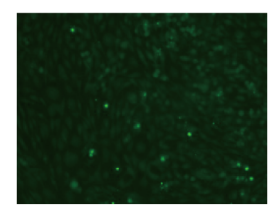

SIM $10^{-8} \mathrm{M}$

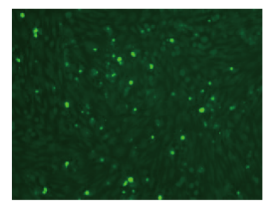

Hcy $5 \mathrm{mM}$

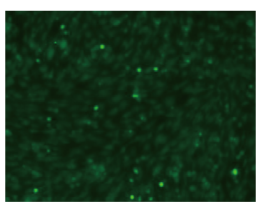

Hcy $5 \mathrm{mM}$ SIM $10^{-9} \mathrm{M}$

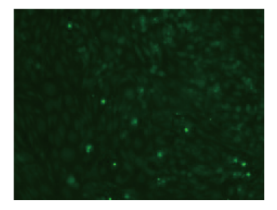

Hcy $5 \mathrm{mM}$ SIM $10^{-8} \mathrm{M}$

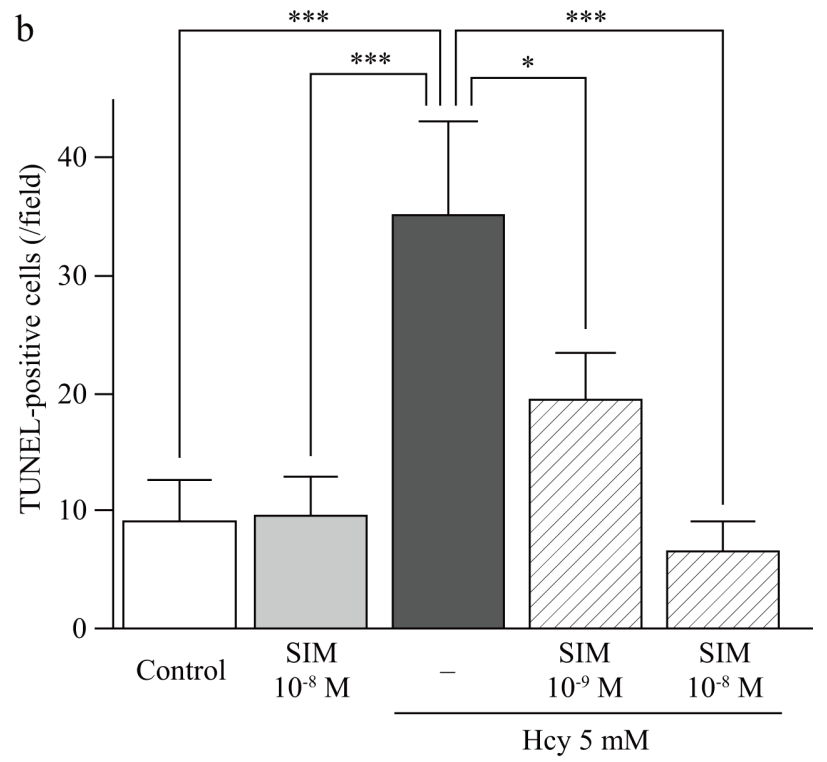

Fig. 1 TUNEL staining of MLO-Y4 cells treated with Hcy and/ or simvastatin

TUNEL staining showed that Hcy-induced apoptosis was suppressed by co-incubation of SIM (a). Quantification of cell count of TUNEL-positive cells showed that coincubation of SIM $10^{-9}$ and $10^{-8} \mathrm{M}$ significantly and dosedependently decreased apoptosis of the cells (b). The result was representative of three different experiments. Results are expressed as the mean \pm standard error of mean (SEM). $* p<0.05$, and $* * * p<0.001$. SIM, simvastatin; Hcy, homocysteine

NADPH oxidases are well-known oxidant enzymes and the dominant source of intracellular oxidative stress. Nox is a complex enzyme composed of two membrane-bound subunits, gp91 ${ }^{\text {phox }}$ and $\mathrm{p} 22^{\text {phox }}$, and four cytosolic subunits, $\mathrm{p} 47^{\text {phox }}, \mathrm{p} 67^{\text {phox }}, \mathrm{p} 40^{\text {phox }}$ and small GTPase, Rac1/2 [19, 20]. Statins have been shown to reduce oxidative stress via regulating expres- 


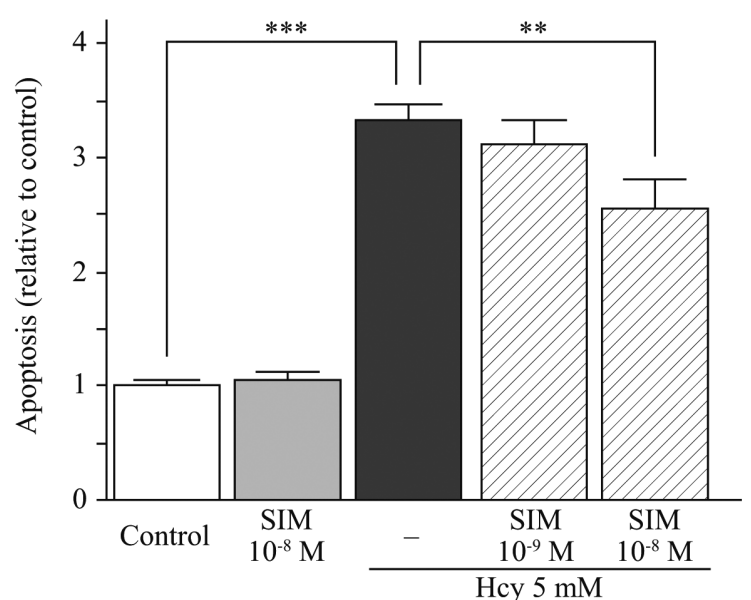

Fig. 2 Apoptosis assay of MLO-Y4 cells with a DNA fragment detection ELISA

DNA fragment detection ELISA analysis showed that Hcy $5 \mathrm{mM}$ significantly increased the apoptosis of MLO-Y4 cells. Hcy's apoptotic effect was suppressed significantly by co-incubation of SIM $10^{-8} \mathrm{M}$. Simvastatin alone had no effect on apoptosis of the cells. The result was representative of three different experiments. Results are expressed as the mean \pm standard error of mean (SEM). $* * p<0.01$, and $* * * p<0.001$. SIM, simvastatin; Hcy, homocysteine.

a

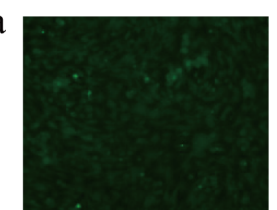

Control

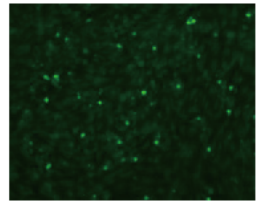

Hcy $5 \mathrm{mM}$

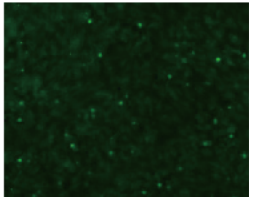

Hcy $5 \mathrm{mM}$

Prava $10^{-8} \mathrm{M}$ b

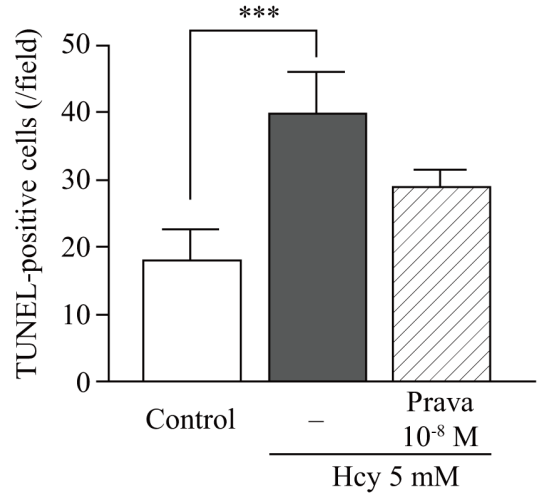

Fig. 3 TUNEL staining of MLO-Y4 cells treated with Hcy and/ or pravastatin

TUNEL staining showed that co-incubation of Prava ameliorated Hcy-induced apoptosis (a). Quantification of cell count of TUNEL-positive cells showed that Prava $10^{-8}$ decreased apoptosis of the cells, however, Prava's effect was not significant (b). The result was representative of three different experiments. Results are expressed as the mean \pm standard error of mean (SEM). $* * * p<0.001$. Hcy, homocysteine; Prava, pravastatin
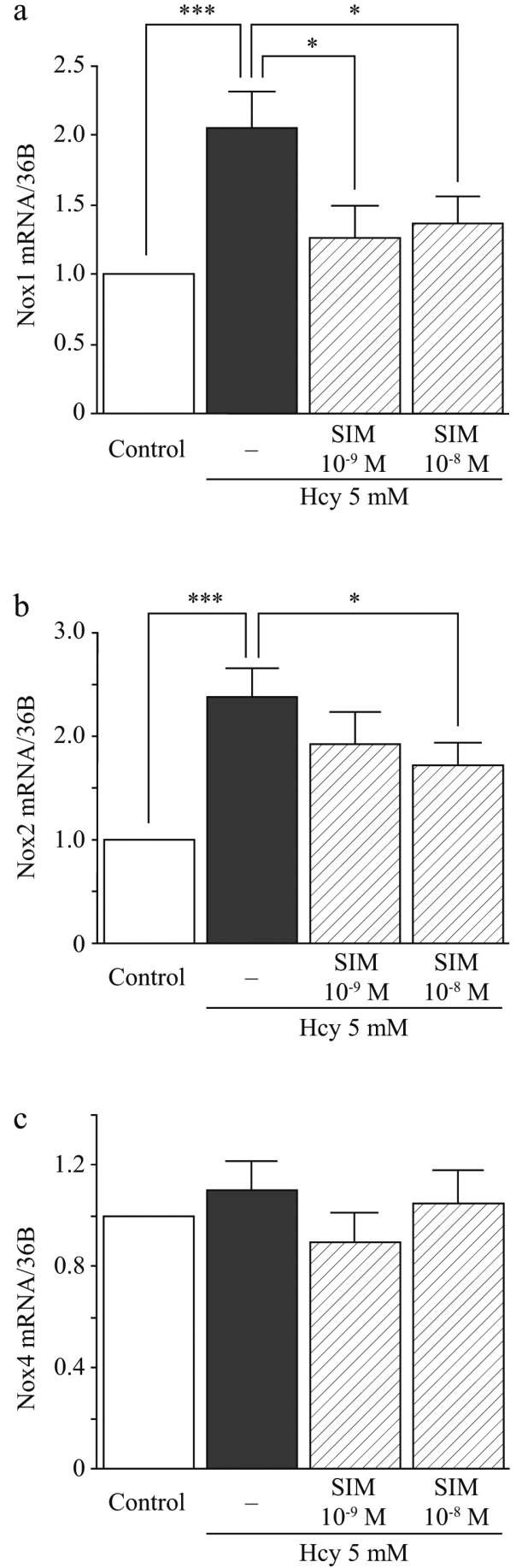

Fig. 4 Effects of Hcy and SIM on expressions of Nox Real-time PCR showed that $5 \mathrm{mM}$ Hcy significantly increased Nox1 (a) and Nox2 (b) mRNA expressions. Co-incubation of SIM $10^{-9}$ and $10^{-8} \mathrm{M}$ significantly decreased the Hcy-induced upregulation of NADPH oxidase 1 (Nox1) (a) and Nox2 (b). The expression of Nox 4 mRNA was not affected by Hcy and SIM treatments (c). Results are expressed as the mean \pm standard error of mean (SEM) $(\mathrm{n}>5) . * p<0.05$, *** $p<0.001$. SIM, simvastatin; Hcy, homocysteine 
sions and membrane translocations of those Nox subunits in various cells and tissues [21-26]. Huang et al. and Yin et al. have shown that treatments with $\mathrm{H}_{2} \mathrm{O}_{2}$ increased Nox4 expressions and induced apoptosis in osteoblastic MC3T3-E1 cells [25] and MG-63 cells [26]. In addition, in their studies, treatment with simvastatin significantly prevented the apoptotic effects of $\mathrm{H}_{2} \mathrm{O}_{2}$ in the cells $[25,26]$. In the present study, we demonstrated the protective effect of simvastatin against Hcy-induced increases in Nox1 and Nox2 expressions and apoptosis of osteocytic cells, suggesting that simvastatin has anti-apoptotic effects on not only osteoblasts but also osteocytes by reducing oxidative stress. In the above studies by Huang et al. and Yin et al., simvastatin reduced the expression of Nox4 stimulated by $\mathrm{H}_{2} \mathrm{O}_{2}$. In contrast, simvastatin ameliorated the expression of Nox 1 and Nox 2 induced by Hcy in our study. Although the mechanism of induction of oxidative stress by Hcy might be different from $\mathrm{H}_{2} \mathrm{O}_{2}$, our findings suggest that the dominant Nox subtypes are Nox 1 and Nox2, but not Nox4, in osteocytes, and that simvastatin regulates Nox1 and Nox 2 expression in the cells.

It is known that the inhibition of the mevalonate pathway induces the differentiation and mineralization of osteoblasts as well as inhibits the activity of osteoclasts and induces osteoclast apoptosis [9]. To our knowledge, this is the first report showing that the inhibition of the mevalonate pathway is important for anti-apoptotic effects in osteocytes. On the other hand, pravastatin did not rescue the Hcy-induced apoptosis of MLO-Y4 cells in this study. There are two types of statin, lipophilic ones, such as simvastatin, and hydrophilic ones, such as pravastatin. Previous studies have shown that lipophilic statins affect osteoblasts and osteoclasts more effectively than hydrophilic ones [6, 27, 28]. Therefore, the effects of lipophilic statins were more potent than those of hydrophilic ones not only in osteoblasts but also osteocytes.

Statins have been shown to exert pleiotropic effects on various cells, which may not be directly associated with cholesterol synthesis. Statins also prevent the synthesis of isoprenoid intermediates such as farnesyl pyrophosphate and geranylgeranyl pyrophosphate as well as small GTP-binding proteins such as Rho, Rac, and Rab, all of which are located downstream of HMG-CoA reductase and have recently attracted widespread attention as the most important factors of pleiotropic effects by statins $[29,30]$. However, the downstream of the inhibition of HMG-CoA reductase in osteocytes is still unknown. Therefore, further studies are needed to determine which downstream molecules are important for the anti-apoptotic effects of statins in osteocytes. On the other hand, we have previously shown that AMPK activation ameliorated Hcy-induced apoptosis of MLO-Y4 cells [18]. Because AMPK is known to inhibit HMG-CoA reductases such as statins [31], the beneficial effects of AMPK might be carried out by the resultant inhibition of the enzyme also in osteocytes. On the other hand, several studies showed that statins activate AMPK in human umbilical vein endothelial cells [32] and neonatal cardiac fibroblasts [33]. Hence, simvastatin might ameliorate Hcy's detrimental effects via AMPK activation. Furthermore, statins have been reported to prevent Nox-induced reactive oxygen species (ROS) and apoptosis via inhibiting phosphorylation of ERK1/2 [22, 23], p38 [23, 34] and JNK [23] and suppression of the NF-kB pathway [23] in other cells and tissues. Therefore, further studies are needed to elucidate the underlying mechanism to statins ameliorative effect on oxidative stress in osteocytes.

In conclusion, the present study demonstrated that simvastatin suppressed the upregulation of Hcyinduced Nox1 and Nox2 and ameliorated Hcy's apoptotic effect in MLO-Y4 cells. These results indicate that the inhibition of the mevalonate pathway by simvastatin may have beneficial effects on excess oxidative stress and apoptosis in osteocytes.

\section{Acknowledgments}

This study received no funding support. Authors' roles: Study design and conduct: AT and IK. Performed the experiments and analyzed the data: AT and MN. Contributed equipment and/or materials: MU, IK, and TS. Wrote the paper: AT and IK. Approved final version: All authors. IK takes responsibility for the integrity of data analysis. The authors wish to thank Dr. Lynda F. Bonewald for providing the MLO-Y4 cell line and Keiko Nagira for technical assistance.

\section{Disclosure}

None of the authors has any conflict of interest to declare. 


\section{References}

1. Shepherd J, Cobbe SM, Ford I, Isles CG, Lorimer AR, et al. (1995) Prevention of coronary heart disease with pravastatin in men with hypercholesterolemia. West of Scotland Coronary Prevention Study Group. $N$ Engl J Med 333: 1301-1307.

2. Downs JR, Clearfield M, Weis S, Whitney E, Shapiro DR, et al. (1998) Primary prevention of acute coronary events with lovastatin in men and women with average cholesterol levels: results of AFCAPS/TexCAPS. Air Force/Texas Coronary Atherosclerosis Prevention Study. JAMA 279: 1615-1622.

3. The Scandinavian Simvastatin Survival Study Group (1994) Randomised trial of cholesterol lowering in 4444 patients with coronary heart disease: the Scandinavian Simvastatin Survival Study (4S). Lancet 344: 1383-1389.

4. The Long-Term Intervention with Pravastatin in Ischaemic Disease (LIPID) Study Group (1998) Prevention of cardiovascular events and death with pravastatin in patients with coronary heart disease and a broad range of initial cholesterol levels. The Long-Term Intervention with Pravastatin in Ischaemic Disease (LIPID) Study Group. N Engl J Med 339: 1349-1357.

5. Palaniswamy C, Selvaraj DR, Selvaraj T, Sukhija R (2010) Mechanisms underlying pleiotropic effects of statins. Am J Ther 17: 75-78.

6. Sugiyama M, Kodama T, Konishi K, Abe K, Asami S, et al. (2000) Compactin and simvastatin, but not pravastatin, induce bone morphogenetic protein-2 in human osteosarcoma cells. Biochem Biophys Res Commun 271: 688-692.

7. Maeda T, Matsunuma A, Kurahashi I, Yanagawa T, Yoshida H, et al. (2004) Induction of osteoblast differentiation indices by statins in MC3T3-E1 cells. J Cell Biochem 92: 458-471.

8. Ohnaka K, Shimoda S, Nawata H, Shimokawa H, Kaibuchi K, et al. (2001) Pitavastatin enhanced BMP-2 and osteocalcin expression by inhibition of Rho-associated kinase in human osteoblasts. Biochem Biophys Res Commun 287: 337-342.

9. Rogers MJ, Crockett JC, Coxon FP, Monkkonen J (2011) Biochemical and molecular mechanisms of action of bisphosphonates. Bone 49: 34-41.

10. Garrett IR, Gutierrez G, Mundy GR (2001) Statins and bone formation. Curr Pharm Des 7: 715-736.

11. Mundy G, Garrett R, Harris S, Chan J, Chen D, et al. (1999) Stimulation of bone formation in vitro and in rodents by statins. Science 286: 1946-1949.

12. Maeda T, Matsunuma A, Kawane T, Horiuchi N (2001) Simvastatin promotes osteoblast differentiation and mineralization in MC3T3-E1 cells. Biochem Biophys Res Commun 280: 874-877.

13. Kaji H, Naito J, Inoue $Y$, Sowa $H$, Sugimoto $T$, et al.
(2008) Statin suppresses apoptosis in osteoblastic cells: role of transforming growth factor-beta-Smad3 pathway. Horm Metab Res 40: 746-751.

14. van Meurs JB, Dhonukshe-Rutten RA, Pluijm SM, van der Klift M, de Jonge R, et al. (2004) Homocysteine levels and the risk of osteoporotic fracture. $N$ Engl J Med 350: 2033-2041.

15. McLean RR, Jacques PF, Selhub J, Tucker KL, Samelson EJ, et al. (2004) Homocysteine as a predictive factor for hip fracture in older persons. $N \mathrm{Engl} \mathrm{J}$ Med 350: 2042-2049.

16. Sato Y, Iwamoto J, Kanoko T, Satoh K (2005) Homocysteine as a predictive factor for hip fracture in elderly women with Parkinson's disease. Am J Med 118: $1250-1255$.

17. Yang J, Hu X, Zhang Q, Cao H, Wang J, et al. (2012) Homocysteine level and risk of fracture: A meta-analysis and systematic review. Bone 51: 376-382.

18. Takeno A, Kanazawa I, Tanaka K, Notsu M, Yokomoto M, et al. (2015) Activation of AMP-activated protein kinase protects against homocysteine-induced apoptosis of osteocytic MLO-Y4 cells by regulating the expressions of NADPH oxidase 1 (Nox1) and Nox2. Bone 77: 135-141.

19. Brown DI, Griendling KK (2009) Nox proteins in signal transduction. Free Radic Biol Med 47: 1239-1253.

20. Lambeth JD, Kawahara T, Diebold B (2007) Regulation of Nox and Duox enzymatic activity and expression. Free Radic Biol Med 43: 319-331.

21. Wassmann S, Laufs U, Muller K, Konkol C, Ahlbory K, et al. (2002) Cellular antioxidant effects of atorvastatin in vitro and in vivo. Arterioscler Thromb Vasc Biol 22: 300-305.

22. Briones AM, Rodriguez-Criado N, Hernanz R, GarciaRedondo AB, Rodrigues-Diez RR, et al. (2009) Atorvastatin prevents angiotensin II-induced vascular remodeling and oxidative stress. Hypertension 54: 142-149.

23. Bruder-Nascimento T, Callera GE, Montezano AC, He Y, Antunes TT, et al. (2015) Vascular injury in diabetic $\mathrm{db} / \mathrm{db}$ mice is ameliorated by atorvastatin: role of Rac1/2-sensitive Nox-dependent pathways. Clin Sci (Lond) 128: 411-423.

24. Zhang F, Sun D, Chen J, Guan N, Huo X, et al. (2015) Simvastatin attenuates angiotensin II-induced inflammation and oxidative stress in human mesangial cells. Mol Med Rep 11: 1246-1251.

25. Huang W, Shang WL, Li DH, Wu WW, Hou SX (2012) Simvastatin protects osteoblast against $\mathrm{H} 2 \mathrm{O} 2$-induced oxidative damage via inhibiting the upregulation of Nox4. Mol Cell Biochem 360: 71-77.

26. Yin H, Shi ZG, Yu YS, Hu J, Wang R, et al. (2012) Protection against osteoporosis by statins is linked to 
a reduction of oxidative stress and restoration of nitric oxide formation in aged and ovariectomized rats. Eur $J$ Pharmacol 674: 200-206.

27. Izumo N, Fujita T, Nakamuta H, Koida M (2001) Lipophilic statins can be osteogenic by promoting osteoblastic calcification in a Cbfa1- and BMP-2-independent manner. Methods Find Exp Clin Pharmacol 23: 389-394.

28. Hanayama R, Shimizu H, Nakagami H, Osako MK, Makino H, et al. (2009) Fluvastatin improves osteoporosis in fructose-fed insulin resistant model rats through blockade of the classical mevalonate pathway and antioxidant action. Int J Mol Med 23: 581-588.

29. Greenwood J, Steinman L, Zamvil SS (2006) Statins in the treatment of central nervous system autoimmune disease. J Neuroimmunol 178: 140-148.

30. Altwairgi AK (2015) Statins are potential anticancerous agents (review). Oncol Rep 33: 1019-1039.
31. Fisslthaler B, Fleming I (2009) Activation and signaling by the AMP-activated protein kinase in endothelial cells. Circ Res 105: 114-127.

32. Sun W, Lee TS, Zhu M, Gu C, Wang Y, et al. (2006) Statins activate AMP-activated protein kinase in vitro and in vivo. Circulation 114: 2655-2662.

33. Hermida N, Markl A, Hamelet J, Van Assche T, Vanderper A, et al. (2013) HMGCoA reductase inhibition reverses myocardial fibrosis and diastolic dysfunction through AMP-activated protein kinase activation in a mouse model of metabolic syndrome. Cardiovasc Res 99: 44-54.

34. Bao XM, Wu CF, Lu GP (2009) Atorvastatin attenuates homocysteine-induced apoptosis in human umbilical vein endothelial cells via inhibiting NADPH oxidaserelated oxidative stress-triggered p38MAPK signaling. Acta Pharmacol Sin 30: 1392-1398. 